\title{
Radicalizar la democracia desde los movimientos sociales. Los casos comparados de Podemos en España y del Frente Amplio en Chile
}

\author{
Radicalizing democracy from social movements. The compared cases of Podemos in Spain and \\ Frente Amplio in Chile.
}

Eduardo Alvarado Espina*, Pablo Rivera-Vargas**, Rommy Morales Olivares ${ }^{* * *}$

\begin{abstract}
Resumen: En este trabajo analizamos las causas que permitieron a los movimientos sociales que emergieron en el año 2011 en España y Chile, transformarse en partidos políticos. Para ello, se recurre a las premisas de la democracia radical referidas a la construcción del otro antagónico y a la noción de partido-movimiento. Desde este marco de referencia se realiza un análisis comparado de los casos de Podemos y el Frente Amplio, considerando cuatro dimensiones explicativas del cambio político: desigualdad socioeconómica; ruptura políticocultural; acciones y discursos de construcción hegemónica; y solidificación política de un quiebre generacional. En base a este análisis es posible constatar, aunque con diferente intensidad, que existieron factores políticos y culturales que permitieron materializar en partidos políticos la dinámica de oposición a la estructura social neoliberal de los movimientos sociales (15M y ME).
\end{abstract}

Palabras clave: Democracia radical, desigualdad, neoliberalismo, partido-movimiento

\begin{abstract}
In this paper, we analyse the causes that allowed social movements in 2011 to transform into political parties in Spain and Chile. For this, an in from the premises of radical democracy regarding the construction of the antagonistic other and the notion of partymovement. From this framework is made a comparative analysis of the cases of Podemos and the Frente Amplio, carried out based on four political change dimensions: socio-economic conditions, political-cultural rupture, actions and discourses of hegemonic construction, and solidification of a generational break. Based on this analysis it is possible to verify, although with different intensity, that political and cultural factors existed that allowed to materialize in political parties the dynamics of opposition to the neoliberal social structure of social movements
\end{abstract}

Keywords: Radical Democracy, Inequality, Neoliberalism, Movement-party

Recibido: 3 junio 2018 Aceptado: 9 agosto 2018

\footnotetext{
* Chileno, Coautor. Doctor en Ciencias Políticas por la Universidad Complutense de Madrid (2017). Consultor e Investigador asociado Departamento de Ingeniería Industrial, Universidad de Chile (Chile). ealvarado@dii.uchile.cl.

** Chileno-Español, Coautor. Doctor en Educación y Sociedad por la Universidad de Barcelona (2014). Académico Departamento de Didáctica y Organización Educativa, Universidad de Barcelona (España). Profesor invitado, Universidad Andrés Bello (Chile), al Doctorado en Educación y Sociedad. Facultad de Educación y Ciencias Sociales. pablorivera@ub.edu

*** Chilena, Coautor. Doctora en Sociología- Socioeconomía y estudios estadísticos por la Universidad de Barcelona (2017). Académica Departamento de Sociología, Universidad de Barcelona (España). Investigadora Postdoctoral. School of Governance. University of the Witwatersrand. (South África). rommy.morales@ub.edu.
} 


\section{Introducción}

La irrupción de movimientos de protesta política durante el año $2011^{1}$ en algunas democracias consolidadas, la cual fue acompañada de una profunda crisis de representatividad de los sistemas políticos, pareció tener como causa común la Gran Recesión de 2008. No obstante, en la etapa postconflicto, este elemento pareció desvanecerse. Poco a poco comenzaron a visibilizarse algunas importantes diferencias entre estos movimientos, sobre todo al intentar constituirse como organizaciones políticas ${ }^{2}$. En algunos casos estos intentos se diluyeron, mientras que en otros fortalecieron alternativas políticas ya existentes. Sin embargo, hubo algunos que inspiraron la creación de nuevos partidos políticos. Esto sucedió con Podemos en España y del Frente Amplio en Chile, que son los casos que se analizan en el presente artículo.

Durante el año 2011, tanto en Chile como en España experimentaron una soterrada crisis de su estructura política post-transicional. Por cierto, un fenómeno que ya ha sido analizado en trabajos anteriores ${ }^{3}$.

Hay que considerar que la Gran Recesión del año 2008 generó un clima propicio para la resistencia social de los estratos sociales más afectados por las medidas económicas regresivas que se adoptaron en varios países. Esto además sucedió en una época marcada por el declive o retroceso de la democracia ${ }^{4}$. Este declive de la política democrática ha sido consecuencia de un proceso de toma de decisiones secuestrado por poderes corporativos, cuya influencia en la política es en gran medida omnímoda. Tal como ha diagnosticado la teoría social y política contemporánea, actualmente la democracia se ha ido reduciendo a sus aspectos formales, diferenciando la economía de la política, y a esta de la sociedad. Con ello, ha terminado consolidándose una versión procedimental y elitista de la misma 5 .

En este contexto, y a partir de las principales características de Podemos en España y del Frente Amplio en Chile, el presente artículo se interroga acerca de cuáles fueron los factores que pudieron incidir para que las demandas de los movimientos sociales del año 2011 cristalizaran en partidos políticos en ambos países.

Para responder a este interrogante, se ha llevado a cabo una rigurosa revisión de antecedentes teóricos y de fuentes de información secundaria relevante. De esa revisión surge un esquema de análisis basado en cuatro dimensiones explicativas del cambio político: (1) desigualdad socioeconómica, (2) ruptura político-cultural, (3) acciones y discursos de construcción hegemónica y (4) solidificación política de un quiebre generacional. Es a partir de este ejercicio que se busca explicar por qué los movimientos sociales del año 2011 provocaron la emergencia y consolidación de Podemos y el Frente Amplio en sus respectivos países.

El trabajo se enmarca teóricamente en el enfoque postestructural de la democracia radical, pues este permite interpretar el descentramiento político vivido en ambos países y cómo se expresó en los movimientos sociales de 2011. Básicamente, se entiende que la política y lo político están en permanente tensión desde los antagonismos de lo social, por lo que no bastaría con una mera descripción institucional

1 Por ejemplo, el 15M en España, la Revuelta en Grecia, Occupy Wall Street en Estados Unidos, el Movimiento Estudiantil en Chile, etc.

2 Íñigo Errejón, "PODEMOS como práctica cultural emergente frente al imaginario neoliberal: hegemonía y disidencia. Conversación con Íñigo Errejón", IC Revista Científica de Información y Comunicación, 11, 2015, 17-46.

3 Patricia Hipsher. "Democratization and the decline of urban social movements in Chile and Spain", Comparative Politics, 28:3, 1996, 273-297.

4 Colin Crouch, Posdemocracia, Madrid, Taurus, 2004; Guy Hermet, El invierno de la democracia, Barcelona, Los Libros del Lince, 2008; Sheldon S. Wolin, Democracia S. A. La democracia dirigida y el fantasma del totalitarismo invertido, Madrid, Katz editores, 2008; Wolfgang Streeck, “The Crisis of Democratic Capitalism”, New Left Review, 71, 2011, 5-29.

5 Eduardo Alvarado Espina, "Una aproximación crítico-contextual al declive de la democracia en la era neoliberal", Revista Española de Ciencia Política, 47, 2018, 69-91. 
para poder escrutar un momento de cambio de ciclo político de disputa hegemónica. En otros términos, el enfrentamiento democrático deja abiertas las posibilidades contingentes para nuevas interpretaciones de lo político, facilitando la configuración de partidos-movimiento con un carácter permanente y procedimental en el juego democrático de cada país.

Finalmente, es relevante resaltar que el presente artículo cuenta con tres importantes potencialidades. La primera, es la utilización de un marco teórico post-estructural que reinterpreta algunos elementos históricos de la etapa post-transicional de los dos países. La segunda, es la realización de un análisis comparado basado en datos secundarios empíricos, tanto cualitativos como cuantitativos. La tercera radica en el propio esquema de análisis comparado, en cuanto se basa en cuatro aspectos que definen las dimensiones políticas de un cambio político, resaltando el pluralismo agonista como elemento constitutivo de la competencia política en las democracias contemporáneas.

El artículo se estructura en cinco apartados. En el primero se presentan las líneas teóricas que se utilizan para el análisis comparativo. En el segundo y tercer apartado se describen el origen y organización de Podemos en España y del Frente Amplio en Chile, respectivamente. En el cuarto apartado se comparan ambos casos mediante la aplicación de las cuatro dimensiones de análisis. Finalmente, se presentan algunas conclusiones y se responde a la pregunta de investigación planteada.

\section{Principales antecedentes teóricos y conceptuales}

\subsection{Democracia radical y la construcción del otro contra-hegemónico}

Hay que partir señalando que se ha estimado que el enfoque postestructural de la democracia radical es el más pertinente para este tipo de análisis. Esta elección se funda en que esta visión propone que la democracia es un régimen político que se construye en torno a la tensión entre lo político y la política. En estos términos, la relación entre los sujetos que se ven afectados por la toma de decisión y los sujetos que deciden es irremediablemente conflictiva y dinámica. Esta relación no se resuelve con la mera aceptación de los procedimientos formales de la democracia representativa.

A pesar de las coincidencias o consensos institucionales de circunstanciales mayorías políticas, lo que hace la idea de la democracia radical es mantener abierta y latente la posibilidad de impugnar las funciones o contenidos del régimen político ${ }^{6}$. Siendo así, bajo este paradigma se podría comprender la tangencial movilidad de contenidos políticos que promueven Podemos y el Frente Amplio en sus respectivos contextos nacionales. Especialmente aquellos que tienen que ver con las pulsiones que subyacen en el tejido social y que no han sido resueltas por la política institucional.

En relación a las instituciones sociales post-transicionales, estas son un conjunto de reglas y recursos relativamente permanentes, que las personas pueden tomar como punto de referencia de las acciones, en tanto impliquen continuidad o cuestionamiento en un contexto de refundación post autoritaria. Aquí, la democracia radical postula la necesaria y persistente subversión de estas reglas como posibilidad de transformación, a través de espacios que permitan una apertura a la interpretación y la diferencia. Este modo de operar potencia el desplazamiento dinámico de las hegemonías y significantes, quedando el poder expuesto a la indeterminación del juego político 7 .

En última instancia, la democracia radical también converge con el acto reivindicativo que define a los movimientos sociales. No hay que olvidar que los movimientos sociales suponen una acción colectiva de resistencia, propuesta y alternativa al orden social instituido y hegemónico, aunque poseen

6 Chantal Mouffe. Desconstrucción y pragmatismo. Buenos Aires, Paidós, 1998; Ernesto Laclau, La Razón populista, Buenos Aires, FCE, 2005; Jacques Derrida. El otro cabo. La democracia, para otro día. Colección Delos. Ediciones del Serval. Barcelona. Pp. 93-94. 1992

7 Mouffe, op.cit. 1998. 
un alto valor subjetivo o heterotópico ${ }^{8}$ Al poseer estas cualidades, pueden ser el germen de una forma primigenia de proyecto político que implique un acontecimiento, producto de una ruptura del consentimiento y la legitimidad de las ideas mayoritariamente aceptadas. Por lo tanto, los movimientos sociales se podrían constituir en un espacio potencial para la cristalización de un discurso contrahegemónico que enfrente al sentido común dominante y al statu quo.

\subsection{Antecedentes y dimensiones del partido-movimiento}

La noción de partido-movimiento no es del todo novedosa. Desde finales del siglo XIX, la democracia moderna ha visto emerger organizaciones de este tipo. De hecho, los primeros partidosmovimiento fueron los partidos de masas, cuyo origen está en el movimiento obrero. Posteriormente, tras la revolución cultural de mayo 68, surgieron otros tipos de movimientos -no materialistas- que también acabaron transformándose paulatinamente en partidos políticos. Esto fue lo que sucedió con el movimiento ecologista en la década de los ochenta. En el primer caso, la emancipación material de la clase obrera fue lo que originó un nuevo tipo de organización política, distinta a los tradicionales partidos de cuadros o de élite del siglo XIX. En el segundo caso, fueron las demandas sectoriales y post-materiales las que dieron auge a nuevas organizaciones políticas que no respondían necesariamente al clivaje de clase. En los casos del 15M español y el Movimiento Estudiantil chileno, el paso a una organización política institucional parece trascender los límites que fija la clase (partidos de masas) y lo monotemático (partidos ecologistas o regionalistas).

Para comprender la emergencia y posicionamiento de Podemos y el Frente Amplio como partidosmovimiento, se establecen cuatro dimensiones de análisis comparativo que apuntan al origen y consolidación de un fenómeno político que surge al margen del consenso racional liberal. Un fenómeno que trasciende los clásicos cánones que marcaron el paso desde los movimientos sociales a partidos políticos.

La primera de estas dimensiones, hace referencia a la desigualdad socioeconómica que afecta a lo político y su implicancia en la estructuración de un espacio contra hegemónico en las heterogéneas sociedades contemporáneas ${ }^{10}$. La segunda, se relaciona con el agotamiento del consentimiento y la legitimidad que otorga la ciudadanía a la dinámica y funcionamiento de las instituciones políticas y a las élites ${ }^{11}$. La tercera se hace cargo del proceso de organización y consolidación institucional desde los movimientos sociales, gracias a acciones y discursos contra-hegemónicos. Por último, la cuarta dimensión se refiere a la instalación de un quiebre generacional.

Ahora bien, estas cuatro dimensiones se acogen a su vez a otras cuatro dimensiones que definen la noción de partido-movimiento que aquí se trabaja. Siguiendo la propuesta de Kitschelt, estas dimensiones serían: 1) condiciones estructurales que impiden a amplios intereses y valores estar representados en las instituciones políticas; 2) una ruptura entre las nuevas demandas de los electores y la disposición de los partidos tradicionales por representarlas; 3) emergencia de partidos que se sustentan en acciones políticas disruptivas y no convencionales; y 4) bajos umbrales institucionales, ya sean formales o informales, que favorecen la consolidación de nuevos partidos ${ }^{12}$.

8 Sidney Tarrow. El poder en movimiento. Los movimientos sociales, la acción colectiva y la politica. Madrid, Alianza, 1997; Alain Touraine y Pablo Rivera-Vargas. “Actores, conflictos y reformas en sociedades de comunicación global”. P. Rivera-Vargas, E. Sánchez, R. Morales-Olivares. (coords). Conocimiento para la equidad social: pensando Chile globalmente. Santiago de Chile, Colección Políticas Públicas - USACH. 2017, 33-40

9 Íñigo Errejón y Chantal Mouffe. Construirpueblo. Hegemonía y radicalización de la democracia, Barcelona, Icaria, 2015.

10 Mouffe, op. cit., 2012; Wolfgang Merkel. "Is capitalism compatible with democracy?”, in Zeitschrift für Vergleichende Politikwissenschaft, 8:2, 2014, 109-128.

11 Bob Jessop. Nicos Poulantzas: Marxist Theory and Political Strategy. Londres, McMillan, 1985.

12 Herbert Kitschelt. "Movement parties". Handbook of party politics, 1, 2006, 278-290. 
De acuerdo a este planteamiento, la acción política transformadora y nueva de los partidosmovimiento sería fruto de un proceso de articulación contra-hegemónico que responde a la contracción de la hegemonía dominante. De esta manera, la relación entre la política (lo social constituido) y lo político (lo constituyente de lo social) se torna en un terreno de disputa del sentido común para fuerzas políticas emergentes, como sucedería en los casos de Podemos en España y el Frente Amplio en Chile. Por lo tanto, el esquema de análisis que se propone brindaría la posibilidad de comparar dos fenómenos políticos de similar alcance, tal como veremos en los siguientes apartados.

\section{Podemos, la organización política de la indignación en España}

\subsection{Antecedentes acerca del origen de Podemos}

Para comprender la génesis de Podemos, hay que remitirse a la crisis económica de 2008 que afectó los tres pilares básicos del Estado de bienestar de la Europa de posguerra (pensiones, educación y salud). La implementación de la política de recortes de gasto público de la Troika, por parte del Gobierno socialista de Zapatero en 2010, llevó a una crisis política que acabó afectando la satisfacción con la democracia en España ${ }^{13}$.

Hay que recordar que la denominada "Europa del corto siglo XX"14, que emergió después de la Segunda Guerra Mundial, tuvo como uno de sus principales desafios la conciliación entre la redistribución de la riqueza y una economía de mercado condicionada por el paradigma socialdemócrata, lo que asentó los estados de bienestar ${ }^{15}$. Sin embargo, esta realidad comenzó a resquebrajarse en los años setenta a partir de la crisis del petróleo, agudizándose en la década de los ochenta. Finalmente, con el fin de la Guerra Fría, en la década de los noventa se produjo una reconfiguración del reparto mundial del poder que favoreció el empoderamiento financiero y aceleró la integración económica capitalista del centro a la periferia. A esto se le llamó globalización, la que actúo como una "correa de transmisión" de la ideología neoliberal como mantra hegemónico en el mundo capitalista.

Este cambio de hegemonía trajo consigo el socavamiento de los cimientos económicos en los que se sostenía la soberanía estatal -política económica y monetaria. A consecuencia de ello se produjo un desmantelamiento de los estados de bienestar y una cesión de la soberanía monetaria a una entidad sin controles democráticos que se concretó en el Tratado de Maastrich ${ }^{16}$.

Otro elemento a considerar es el pasado político inmediato de España. El ocaso de la dictadura franquista (1939-1975), dio paso a una transición política -también económica- que tuvo dos importantes características. En primer lugar, se trató de un proceso político marcado por la crisis del petróleo (y sus consecuencias a nivel mundial) y por la fuerte movilización democrática contra la dictadura. En segundo lugar, el cambio político fue liderado por las élites políticas y económicas configuradas durante el franquismo ${ }^{17}$. Esta realidad generó estabilidad al régimen democrático resultante. No obstante, en las últimas dos décadas surgieron procesos y movilizaciones cuyos objetivos fueron: recuperar el poder soberano del pueblo, promover procesos de memoria histórica y limitar el poder de las finanzas. Unos que fueron objetivos acompañados de elementos más radicales y nuevas significaciones de la política.

13 Centro de Investigaciones Sociológicas, 2013.

14 Eric Hobsbawm. Guerra y paz en el siglo XXI, Barcelona, Crítica, 2007.

15 Gøsta Esping-Andersen. Politics against markets: The social democratic road to power, Princeton, NJ, Princeton University Press, 1985.

16 El "Tratado de Maastricht", constituye una piedra angular en el proceso de integración europea, pues por primera vez se sobrepasaba el objetivo económico inicial de la Comunidad (construir un mercado común) y se le da una vocación de unidad política. Consagra oficialmente el nombre de "Unión Europea" que en adelante sustituirá al de Comunidad Europea.

17 El origen de Alianza Popular -que después pasaría a denominarse Partido Popular- es un buen ejemplo de esta realidad. El partido fue fundado por ministros y cargos más que relevantes del aparato franquista. Además, se crearon entidades financieras y grandes empresas cuyo crecimiento estuvo vinculado a este régimen (Monedero, 2012). 


\subsection{La crisis de régimen y la emergencia del $15 \mathrm{M}$}

En España, tanto la crisis económica del año 2008 como a las políticas de austeridad adoptadas por el Gobierno, apoyadas por la élite económica, provocaron una crisis de régimen ${ }^{18}$. Estas políticas sellaron el camino hacia una profundización neoliberal con la posterior llegada del Partido Popular al Gobierno en 2011. Todo ello hizo inevitable la emergencia de nuevas posiciones contra-hegemónicas en el espacio político, originando al mismo tiempo una pérdida de legitimidad de los sectores dominantes. El principal indicador fue la pauperización de las condiciones de vida de las llamadas clases medias -precarización del trabajo y pérdida de derechos sociales. Un grupo social fundamental para el equilibrio entre estructura económica y democracia. Este hecho trajo consigo un quiebre de los consensos que articulaban la vida en común.

Según Monedero ${ }^{19}$ se trató de una crisis de régimen, que se develó en cuatro dimensiones. En primer lugar, una ruptura de los principales consensos que permitían articular la vida en común. En segundo lugar, emergencia de contradicciones y fracturas en el bloque dominante, lo que quedó en evidencia, por ejemplo, en los casos de corrupción ${ }^{20}$. En tercer lugar, las políticas de austeridad que se ensañaron con la precaria situación social de los sectores populares, básicamente mediante recortes del gasto público, desempleo, pérdida de poder adquisitivo, y disminución de libertades civiles y políticas ${ }^{21}$. Finalmente, en cuarto lugar, en un cambio del sistema de partidos justo después del estallido del $15 \mathrm{M}$ en 201122, que supuso una ruptura de la lógica bipartidista.

En este contexto, el movimiento 15M fue la respuesta y expresión social por excelencia de la crisis de régimen ${ }^{23}$. Prueba de ello es que según los datos del CIS, el 80\% de la población española apoyó sus demandas ${ }^{24}$. El también llamado "Movimiento de los Indignados", nació en la manifestación del 15 de mayo de 2011, con la acampada de cuarenta personas en la Puerta del Sol de Madrid. Desde aquel momento, este modo de protesta se serializó en todo el territorio español, masificando y replicando sus demandas de democracia más participativa y alejada del bipartidismo PSOE-PP; y fuera del imperio de bancos y corporaciones ${ }^{25}$. Por estos motivos, para los fundadores de Podemos, el 15M inauguró una nueva gramática política y selló las bases de construcción de un nuevo sentido común de época, una nueva articulación de los consensos sociales para organizar la vida en común ${ }^{26}$.

\subsection{De movimiento a partido o la nueva forma de hacer política de Podemos}

De acuerdo a Íñigo Errejón, el 15M abrió una "ventana de oportunidad" para desafiar la hegemonía. Esto es, un espacio para la diferencia, la novedad radical y la emergencia de un actor que fuera capaz de representar, en términos gramscianos, un nuevo sentido común de época. En este caso, la voluntad de reconstruir ciertos consensos quebrantados por las élites políticas del bipartidismo y que, al

18 Juan Carlos Monedero. "¿Posdemocracia? Frente al pesimismo de la nostalgia, el optimismo de la desobediencia”, Nueva Sociedad, 240, julio-agosto, 2012.

19 Monedero, op. cit, 2012, 68-86.

20 Manuel Villoria y Fernando Jiménez. “La corrupción en España (2004-2010): datos, percepción y efectos”, Revista Española de Investigaciones Sociológicas (REIS), 138:1, 2012, 109-134.

21 Vicenç Navarro. "El error de las políticas de austeridad, en la sanidad pública", Gaceta Sanitaria, 26: 2, 2012, 174-175.

22 En las elecciones generales del año 2011, el Partido Popular gana con mayoría absoluta, y el PSOE pierde el 40\% de sus apoyos electorales.

23 Manuel Castells, Redes de indignación y esperan₹a, Madrid, Alianza, 2012.

24 Centro de Investigaciones Sociológicas (CIS). Barómetro Mayo 2014. Estudio 3024. Madrid: CIS. 2014.

25 Así como una auténtica división de poderes y otras medidas con la intención de mejorar el sistema democrático (Castells, op.cit. 2012).

26 Pablo Iglesias, “Understanding Podemos”, in New Left Review, 93, 2015, 7-22; Errejón, op.cit. 2015- 
mismo tiempo, fuera capaz de constituirse en una herramienta política que incorporara a los desilusionados y desafectos del sistema de partidos tradicional.

Podemos irrumpió en el espacio de la política con la difusión del manifiesto "Mover ficha: convertir la indignación en cambio político", en enero de 2014. Este manifiesto expresó la necesidad de crear una lista del partido para competir en las elecciones al Parlamento Europeo de ese año, con el objetivo de oponerse a las políticas dominantes de la UE. Oficialmente, Podemos se fundó el 16 de enero de 2014 en el Teatro del Barrio de Madrid. Desde un comienzo, su equipo promotor trabajó con la hipótesis de poder articular, en base a la nueva gramática del $15 \mathrm{M}$, las mayorías populares para hacer frente a las élites ${ }^{27}$. Esto se llevó a la práctica a partir de un liderazgo mediático como herramienta aglutinadora, constructor de sentido y referencia común de la diversidad de actores que se sumaron a la construcción del espacio político de cambio $^{28}$. Con Podemos, España ingresó definitivamente en la era de la "democracia de audiencias"29.

Su primer hito político de calado fue conseguir más de un millón doscientos mil votos, cinco diputados y ser la tercera fuerza política en 23 de las 40 ciudades más grandes de España en las elecciones al Parlamento Europeo realizadas en mayo del $2014^{30}$. A partir de este instante, Podemos comienza a transformarse en la expresión política por excelencia de la crisis de régimen, lo que se consagra con el segundo gran hito, la obtención de más de cinco millones de votos en las elecciones generales de 2015, y de 71 diputados en el Parlamento Español ${ }^{31}$.

En síntesis, se puede decir que Podemos fue adoptando la forma de un partido-movimiento, al mismo tiempo que participaba de las contiendas electorales como un partido vertical y con voluntad de poder. De este modo, combinó la lógica de trabajo de un movimiento con la participación política activa en el sistema político. Su objetivo, que la sociedad civil utilizara esta herramienta de maneras muy diversas para participar de la vida pública.

\section{Frente Amplio, la emergencia de una izquierda hegemónica en Chile}

\subsection{Antecedentes y contexto histórico}

Las movilizaciones en Chile del año 2011 tuvieron un gran impacto en la nueva configuración política del país. A juicio de Garretón ${ }^{32}$, expresaron una ruptura entre la política clásica y la sociedad civil, de un modo equivalente a las de Mayo 68`en Europa o a las de Chiapas del año 1994 en América Latina.

En la década de los ochenta Chile experimentó uno de los períodos de desarrollo capitalista más acelerados de la historia global ${ }^{33}$, lo que por otra parte significó el desmantelamiento del proceso de transformación social del gobierno socialista de Salvador Allende (1970-1973). Tras el golpe de Estado de 1973, la dictadura cívico-militar impulsó reformas estructurales de corte neoliberal que aumentaron la desigualdad. Se fomentó la privatización del tejido productivo del país, además de confiar el desempeño de toda la economía al mercado ${ }^{34}$.

27 Pablo Iglesias, Íñigo Errejón, Juan Carlos Monedero, Carolina Bescansa y Luis Alegre. Borrador de Principios Políticos, Madrid, Podemos. 2014. Recuperado de: goo.gl/C0p52D

28 Iglesias, op. cit., 2015.

29 Bernard Manin. Los principios del Gobierno Representativo. Madrid, Alianza, 2010.

30 Parlamento Europeo. Resultado elecciones europeas de 2014, 2014, Recuperado de goo.gl/hjhXi3

31 Congreso de los Diputados, Resultados elecciones generales, 2015. Recuperado de: goo.gl/scnDIQ

32 Manuel Antonio Garreto $\square$ n. La gran ruptura: Institucionalidad politica y actores sociales en el Chile del siglo XXI. Santiago, LOM ediciones, 2016.

33 Ricardo Ffrench-Davis. Entre el Neoliberalismo y el Crecimiento con Equidad: Tres D $\square$ cadas de Pol $\square$ tica Econo $\square$ mica en Chile, Santiago de Chile, LOM Ediciones, 2003.

34 Patricio Rozas y Gustavo Marín. “1988: El mapa de la extrema riqueza 10 años después”, CESOC, Santiago de Chile, 1988. 
La idea "refundacional" de la oligarquía económica y los militares, en palabras de uno de los principales ideólogos del régimen, Jaime Guzmán ${ }^{35}$, la "nueva economía” sería un sistema que permitiría superar el "estatismo exagerado" 36 . Esta premisa fue la base de la comprensión dictatorial. Las reformas se llevaron a cabo desde el Estado, diseñando una nueva arquitectura de un sistema económico paradójicamente liberalizado por ese mismo Estado.

Con el advenimiento democrático en el año 1990, Chile mantuvo el sustrato de estas reformas. No hubo intervención del Estado alguna, no obstante los graves problemas de deuda social ${ }^{37}$, la inflación descontrolada y la exacerbada apertura económica ${ }^{38}$. De este modo, el modelo económico de la Concertación -continuado por la Nueva Mayoría ${ }^{39}$ - no hizo frente a la redistribución, solidificando la realidad social construida por la ideología neoliberal. Las primeras manifestaciones públicas de disconformidad respecto a estas políticas se evidencian al final del gobierno de Patricio Aylwin (1990-1994), volviéndose más radicales en la primera etapa del gobierno de Michelle Bachelet (2006-2010).

Fue al comienzo de los años noventa cuando el nuevo gobierno democrático pudo elegir entre un cambio sustancial en la estrategia económica - de corte más socialdemócrata- o gestionar el modelo económico neoliberal en un marco institucional condicionado por "enclaves autoritarios" 40 . Ya fuese por los chantajes del gran empresariado o las amenazas de unas fuerzas armadas corporativistas y adeptas al ex dictador Pinochet, acabaron aceptando lo heredado, limitándose a hacer ajustes considerados básicos para una mayor eficiencia económica y una mayor igualdad social.

\subsection{La crisis de la política institucional: la estructura social cuestionada por el movimiento estudiantil}

En la primera década del siglo XXI se comenzó a vivenciar la insalvable brecha social que dejó la continuidad de la arquitectura económica neoliberal de la dictadura. Este hecho tuvo dos grandes consecuencias. Por un lado, su naturalización y penetración en las conductas sociales. Esto se constata en la adaptación de la ciudadanía a los valores promovidos por el modelo que llevan a una extrema individualización. Por otro lado, el ascendente distanciamiento de la sociedad respecto a la política institucional. En este escenario es donde emerge el movimiento estudiantil de 2011, cuya principal demanda fue una educación pública, gratuita y de calidad. Este tipo de demanda fue fundamental para los cambios que se comenzarían a percibir posteriormente en la política, pues implicó un antes y un después en el modo de plantear las reformas desde los movimientos sociales. Esto debido a que esta no atiende a una estrategia basada en la "propuesta mesurada y acordada" que promovía la cultura de los consensos de las élites políticas de la transición, sino que a una verbalización de un acto radical y rupturista. Asimismo, fue el impulso y apoyo mayoritario que consisto esta demanda en la sociedad chilena, lo que hizo visible a los grupos políticos que fueron una parte fundante de este movimiento, como Revolución Democrática (RD) ${ }^{41}$ y el Movimiento Autonomista (MA).

35 Arturo Fontaine. "El miedo y otros escritos: el pensamiento de Jaime Guzmán”, en Estudios Públicos, 42, 1991.

36 Según Guzmán (en Fontaine, op. cit, 1991), el estatismo: "perturba el crecimiento sano y acelerado de la economía, una institucionalidad concebida al servicio de la libertad y el progreso debe robustecer una economía libre, sin la cual la democracia política puede terminar reduciéndose a una fórmula hueca" (pp.336)

37 Carlos Durán, “Campo político-institucional y procesamiento del malestar social en Chile, 1999-2009”, Izquierdas, 40, 2018, $1-32$.

38 Rommy Morales-Olivares. "Inconsistencies between social-democratic discourses and neo-liberal institutional practices in Chile and South Africa: a comparative analysis of the post-authoritarian periods". Peter Wagner, P. (Ed). African, American and European trajectories of modernity, in Edinburgh, Edinburgh University Press, 2015, 125-148.

39 La Concertación de Partidos por la Democracia fue la coalición que gobernó en Chile desde 1990 a 2010. A partir de 2011 pasó a denominarse Nueva Mayoría (2010-2018). Su élite política proviene, en su gran mayoría, de los partidos perseguidos durante la dictadura chilena (1973-1990).

40 Garreto $\square$ n, op cit. 2016.

41 El partido Revolución Democrática (RD) fue fundado el 7 de enero de 2012 por algunos de los líderes de la movilización 
El año 2016 estos movimientos políticos anunciaron la construcción de un Frente Amplio. Una coalición con la que se compitió en las elecciones presidencial y parlamentaria de 2017, con el fin de terminar con el reparto bipactista del poder entre Nueva Mayoría (ex Concertación) y la alianza de derechas Chile Vamos. Ahora bien, el objetivo principal -y radical-con el cual se presenta el Frente Amplio a estas elecciones, fue el de superar las causas estructurales de la desigualdad.

Cabe mencionar que el primer acontecimiento que explicita la fisura del consenso elitista en torno al modelo de desarrollo que impulso la emergencia de Frente Amplio se originó en los movimientos sociales del año 2006, con la llamada "revolución pingüina"42, que fue desactivada y procesada por la ex Concertación y la derecha. A pesar de ello, terminó por provocar la explosión del gran movimiento estudiantil del 2011. Existe una explosión tanto de masividad como de creatividad, donde la performance política comienza a ser un método común en las movilizaciones. Teniendo en cuenta estas manifestaciones, Revolución Democrática y el Movimiento Autonomista propusieron un estado de movilización continua, desde el cual conseguir ser una expresión política que aspirara al poder institucional.

El movimiento estudiantil del 2011 sorprendió a las élites políticas tradicionales, incluyendo a la opinión pública en general, ya que introdujo una nueva gramática política a las demandas sociales. La movilización se originó primero en la educación superior privada. Aquellos estudiantes que accedieron a estudios superiores a través de un sistema de crédito bancario con garantía estatal, conocido por sus siglas $\mathrm{CAE}^{43}$, protestaron masivamente por una forma de pago abusivo y estigmatizante para los jóvenes de estratos sociales bajos, que son los supuestos "beneficiarios" de la mayor cobertura del sistema universitario. Un reclamo que se extendió a todo el sistema universitario, dando legitimidad a jóvenes líderes políticos ${ }^{44}$ que promovían el final de la privatización (financiación) del sistema educativo, especialmente en su nivel superior.

\subsection{El movimiento estudiantil, retórica y dinámica del Frente Amplio}

Una de las metas que se propuso el Frente Amplio fue la construcción de puentes entre el movimiento social y la organización partidista, apuntando a la unidad de acción. Para hacerlo, esta coalición política contó en su haber con la épica y el relato del movimiento estudiantil. Su primer gran hito político se produjo durante la primera mitad del año 2016, donde se gestó un movimiento social comunal que consiguió un bullado éxito al ser elegido como Alcalde de Valparaíso el ex líder estudiantil Jorge Sharp, en las elecciones del 23 de octubre del año 2016.

La propuesta retórica del Frente Amplio acabó generando una ruptura radical en el espacio político de Chile, ya que la izquierda ubicada al margen de los consensos de la transición se ha definido como extraparlamentaria y -desde 1973- no ha contado con presencia en el poder institucional, salvo la del Partido Comunista de Chile tras su incorporación a la Nueva Mayoría en 2010. Ahora bien, el Frente Amplio se aleja del PC, al atribuirse en su discurso la representación de los compromisos políticos de los movimientos sociales.

A groso modo, el Frente Amplio se autodefinió en un comienzo como un conglomerado heterogéneo capaz de aunar movimiento social y política institucional. Al igual que Podemos, denunció

estudiantil de 2011, como el actual diputado Giorgio Jackson. Se define como un movimiento político de izquierda que busca la construcción de un socialismo democrático y la democracia participativa.

$42 \mathrm{El}$ movimiento pingüino se llevó a cabo en el año 2006 por los estudiantes de educación secundaria. Fue considerada la primera movilización social masiva y de carácter nacional en Chile desde la recuperación de la democracia. Su principal reivindicación fue la educación pública y de calidad en educación secundaria y superior.

43 El CAE es un crédito para financiar estudios de educación superior a los chilenos y que debe pagarse por un tiempo mínimo de 10 años.

44 Entre los más destacados: Camila Vallejos (Partido Comunista), Gabriel Boric (Movimiento Autonomista), y Giorgio Jackson (Revolución Democrática), los tres dirigentes estudiantiles en las movilizaciones del 2011. 
el problema de la desigualdad y sus justificaciones ideológicas, además de diseñó y consolidó otras demandas que han de ser resueltas ${ }^{45}$. Además, se atribuyó la representación de las clases subalternas.

Así, desde el movimiento social se cristaliza una nueva hegemonía no ajena a la conflictividad propia de una visión democrática radical. Es decir, de la tensión entre representación de las intenciones y convicciones, en un marco de extrema institucionalización y profesionalización de la actividad política. Esta dinámica significó seguir profundamente vinculado, inmerso e implicado en los movimientos sociales respetando su autonomía, para dejar abierta la ventana a nuevas formas de resolución contingente y contra hegemónicas.

\section{4.- Una aproximación comparada a los nuevos actores políticos}

Los movimientos sociales pueden ser un espacio para radicalizar la democracia a partir de las brechas entre lo politico y la política que estos representan. Esta posibilidad engarza con el enfoque postestructural de la democracia, en tanto mantiene abierta la posibilidad de renovación de los componentes del régimen político, contraviniendo a su vez su solidificación elitista. Ese es el espacio de impugnación hegemónica desde lo político que propone la democracia radical.

Desde esta perspectiva, existe una serie de condiciones que pueden viabilizar la organización de nuevas hegemonías. Algunas de estas condiciones son: que el poder económico quede expuesto a una indeterminación redistributiva; que las decisiones vinculantes estén abiertas a lo indecidible ${ }^{46}$ en términos normativos, culturales y con el pasado; que la génesis de las nuevas hegemonías no se vean limitadas; y que por particularista que sea una visión subversiva de la realidad, opere de modo universalizante sin que su proyecto político se convierta en totalitario ${ }^{47}$.

Teniendo en cuenta estas premisas, a continuación, se realiza un análisis comparativo de las circunstancias que explicarían la emergencia y consolidación de Podemos y el Frente Amplio como partidos-movimiento capaces de representar un otro antagónico y hegemónico. Para este afán, se utiliza un esquema de análisis a partir de cuatro factores: 1) desigualdad socioeconómica; 2) ruptura políticocultural; 3) acciones y discursos de construcción hegemónica; y 4) solidificación política de un quiebre generacional.

\subsection{Desigualdad socioeconómica}

Las condiciones socioeconómicas en España y Chile, cuando se produjo el estallido social de 2011, son divergentes. Según datos de la OCDE48, en España la desigualdad social se incrementa tras la crisis económica de 2008, mientras que en Chile se reduce, debido principalmente a la bonanza que generó el precio del cobre y las políticas anti-cíclicas del primer gobierno de Michelle Bachelet (2006-2010). Sin embargo, las diferencias estructurales siguen siendo ostensibles, como lo refleja el coeficiente Gini ${ }^{49}$, que fue de 0,53 puntos en Chile y 0,34 en España entre 2008 y 201450. Asimismo, el nivel de bienestar de España es superior al de Chile, a pesar de la implantación de medidas económicas regresivas para la distribución del ingreso (aumento del IVA y recortes sociales) por parte del gobierno del Partido Popular.

45 Gabriel Boric. "Los movimientos sociales y la política de hoy: una mirada con perspectiva histórica”, Pablo Rivera-Vargas, Elena Sánchez, Rommy Morales-Olivares, (coords). Conocimiento para la equidad social: pensando Chile globalmente. Santiago de Chile, Colección Políticas Públicas USACH, 2016, 250-256.

46 Mouffe, op cit. 1998.

47 Laclau, Ernesto. 1998. Deconstrucción, pragmatismo y hegemonía. En Mouffe, Chantal. (comp). Deconstrucción y Pragmatismo. Buenos Aires. Paidós.

48 OCDE, ¿Cómo va la vida en España? Better Life Iniciative. Paris: OCDE, 2016, Recuperado de: goo.gl/FXHbWJ

49 Indicador que mide la desigualdad dentro del país entre 0 y 1

$50 \mathrm{El}$ índice Gini promedio en los países de la OECD es de 0.31 en este mismo período, tres puntos inferior al de España y 0.22 más bajo que el de Chile. 
Otro elemento importante en la comparación socio-económica, es el gasto en tres áreas del bienestar social: educación, salud y pensiones. Un tipo de gasto que favorece la transferencia de ingresos de ricos a pobres ${ }^{51}$. Este elemento es importante debido a que unas malas condiciones de vida pueden afectar negativamente el valor de la democracia y su calidad ${ }^{52}$. En la Tabla 1 se aprecia el gasto realizado en cada una de estas áreas en el período 2008-2011, que muestra una significativa diferencia entre España y Chile.

Tabla 1 Porcentaje de gasto social en España y Chile, 2008-2011

\begin{tabular}{l|lccccc} 
País & Tipo de gasto & 2008 & 2009 & 2010 & 2011 & Media \\
\hline \multirow{3}{*}{ Chile } & Educación & 3,8 & 4,2 & 4,2 & 4,1 & 4,1 \\
& Salud & 6,9 & 7,4 & 7 & 7 & 7,1 \\
& Pensiones & 3,3 & 3,5 & 3,4 & 3,2 & 3,4 \\
\multirow{5}{*}{ España } & Educación & 4,5 & 4,9 & 4,8 & 4,8 & 4,8 \\
& Salud & 8,8 & 9,5 & 9,6 & 9,5 & 9,4 \\
& Fuente: elaboración propia & 8,6 & 9,5 & 10 & 11 & 9,7 \\
\hline
\end{tabular}

La desigualdad estructural en Chile y el aumento de la misma en España a partir de 2009, creó unas condiciones que aumentaron el malestar y disconformidad hacia el sistema político, en tanto gestor y reproductor de estas condiciones. Además, el escaso gasto público en áreas básicas del bienestar brindó un marco de acción y de justificación a toda movilización que promoviera desafiar el statu quo. En otras palabras, el aumento -o la invariabilidad- de las brechas económicas y sociales constituyó un momento propicio para reivindicar los antagonismos, desde lo social a lo político, como sugiere la concepción de democracia radical.

Por lo tanto, la instalación de una realidad económica y social que precarizó la vida de gran parte de la clase trabajadora en España o la profundización de dicha realidad en Chile fue el detonante de un momento de cierre de un ciclo político y apertura de uno nuevo. Como entendieron los futuros dirigentes de Podemos y del Frente Amplio, ese momento de legitimación de los movimientos sociales brindó la oportunidad de articular políticamente una contra hegemonía al relato político e ideológico neoliberal. Se inauguró una nueva dialéctica para impugnar a la ideología dominante.

\subsection{La ruptura político-cultural del consenso de las élites}

Chile y España tienen una cultura política marcada por el desinterés y la desafección desde hace décadas ${ }^{53}$. A esto se añade una escasa confianza, tanto interpersonal como hacia las instituciones políticas $^{54}$. Esta carencia de capital social no parece cuestionar la legitimidad de la democracia en ninguno

51 Thomas Piketty, El capital en el siglo XXI. Barcelona, RBA, 2015.

52 Merkel, op. cit. 2014.

53 De acuerdo a la sexta ola de la World Values Survey (2010-2014), en España sólo el 19\% de los individuos declara confiar en los demás, en Chile el porcentaje se reduce al $12 \%$.

54 Pablo Rivera-Vargas, "Modernización Reflexiva en Chile: Participación Electoral y Confianza de la Ciudadanía en las Instituciones durante la Post Dictadura: Análisis entre los Años 1999-2010”, Políticas Públicas, 9:2, Santiago, Universidad de Santiago, 2016, 90-108. 
de los casos -quizás una especie de cinismo político. La ciudadanía española apoya la democracia mayoritariamente, a pesar de la crisis económica y política que ha vivido el país desde 2008. De hecho, hacia 2013 el 75\% de la población señalaba que este régimen político era el mejor para España ${ }^{55}$. Por su parte, el 63\% de los chilenos señaló apoyar la democracia ese mismo año ${ }^{56}$. En otros términos, no fue la democracia, ya sea como concepto o ideal político, lo que entra en crisis en estos países. Por tanto, hay que indagar en una de las principales dimensiones de la democracia representativa, el sistema de partidos.

La frase que más resonó en España en la primavera de 2011, fue aquel "no nos representan", que el 15M dedicó a las élites políticas y los partidos tradicionales. En otras palabras, gran parte de la indignación de la ciudadanía estuvo dirigida contra lo que se conoce como partidocracia o democracia de partidos. Este argumento se sostiene en dos hechos. Por una parte, los dos partidos que han gobernado indistintamente España desde 1982, "acabaron funcionando como partidos omnímodos y exentos de control, cada vez más desconectados de la sociedad civil, debido a su arraigada tendencia a la endogamia y la cooptación" 57 . Por otra, la dinámica de confrontación política monopolizada por el bipartidismo develó su artificialidad a causa de las medidas de austeridad fiscal acordadas por PSOE y PP para enfrentar los efectos de la crisis económica en 2010.

Algo similar sucedió en el caso del sistema de partidos en Chile. El movimiento estudiantil verbalizó el decreciente apoyo que tenían los partidos y coaliciones que venían gobernando el país desde 1990. La generación de universitarios que lideró este tercer ciclo de protestas estudiantiles ${ }^{58}$ consiguió dar cobertura tanto a los ciudadanos desafectos del sistema político como a aquellos más politizados y críticos con el mismo. Ante ello, el sistema de partidos tradicional se tensionó en un movimiento contradictorio. Algunos partidos intentaron incorporar las demandas del movimiento estudiantil -incluso cooptar a los nuevos dirigentes-, al tiempo que otros buscaron refugiarse en la extemporánea "política de los consensos" de las élites políticas y económicas de la transición.

Por último, el creciente apoyo a los valores post-materiales -igualdad de derechos, ecologismo, tolerancia ${ }^{59}$ - en ambos países, conectó culturalmente a estos nuevos actores políticos con una parte importante de la ciudadanía que se expresaba en otros movimientos menos generalistas. Se produjo un espacio de confluencia con otras reivindicaciones colectivas (igualdad de género, diversidad sexual, ecologistas).

La ruptura con la dialéctica política transicional, la estructura de oportunidades políticas, así como los contextos de interacción y movilización de la contienda en cada caso ${ }^{60}$, se legitimó. Para más abundamiento, esto puso en relieve un fuerte rechazo a los respectivos sistemas de partidos. Todo esto derivó en una legitimación de los espacios extra-institucionales que desafiaban la acción de la política tradicional, exigiendo más democracia.

\subsection{Acciones y discursos de construcción hegemónica}

Una característica que comparten Podemos y el Frente Amplio en su emergencia, es que sus procesos de construcción hegemónica son desencadenados tras la acción colectiva de potentes movimientos sociales. Un hecho que por lo demás encaja dentro de las formas de participación política

55 Este porcentaje es menor al 81\% previo a la crisis económica de 2008.

56 Latinobarómetro, op. cit. 2013.

57 Juan Jesús González, "Crisis de la democracia de partidos y segunda transición”, Revista de Derecho Político, 100, 2017, 615-638.

58 Luis Thielemann. "Para una periodificación del Movimiento Estudiantil de la transición (1987-2011)", Revista Pretérito Imperfecto, 2011.

59 World Values Survey. Wave 6, 2010-2014. Viena, Institute for Comparative Survey Research, 2014. Recuperado de: https://goo.gl/Cz74Bd

60 Pablo Oñate, "La movilización ciudadana en España en los albores del siglo XXI: una contextualización para el debate", Revista Española de Ciencia Política, 33, 2013, 31-55. 
que suelen identificarse con el cambio cultural de la era posindustrial ${ }^{61}$. Proceso de los cuales suelen surgir nuevos actores, redes formales e informales de movilización, que van en perjuicio de las clásicas élites y los vehículos tradicionales para la participación ${ }^{62}$. En estos casos además se consolidan como un actor político procedimental, gracias a un diseño organizacional relativamente permanente como es el del partido-movimiento ${ }^{63}$.

En una primera etapa, Podemos surge de la red de complicidades que generó el 15M entre militantes de partidos minoritarios de izquierdas y líderes de organizaciones sociales. Esta primigenia organización -el núcleo promotor-se vio posteriormente reforzada por un proceso auto-convocado para la creación de "círculos ciudadanos" en toda España. Una movilización de voluntades que nació de una entusiasta auto-convocatoria ciudadana. A partir de estas acciones organizativas, Podemos fue paulatinamente convirtiéndose en un partido-movimiento que se contrapuso a los partidos cartel de la última época ${ }^{64}$.

Por su parte, el Frente Amplio se organizó, principalmente, a partir de la confluencia de partidos provenientes de la izquierda extra-institucional chilena y pequeños movimientos políticos de corte universitario. Este conglomerado no se vinculó exclusivamente a esos movimientos sociales. Y aunque en un comienzo no contó con una organización territorial similar a la de Podemos en España ni con el impacto que este partido obtuvo tras su irrupción en las elecciones europeas de 2014, si pudo constituirse en un referente en algunos territorios que permitieron su posterior consolidación institucional.

En su fase de construcción, los dos partidos apelaron discursivamente a la gente -al pueblodiferenciándose de las élites políticas convencionales. Esta estrategia resultó útil para solventar dos problemas que hacen difícil la acción contra-hegemónica, como la sofisticación de la política en códigos de expertos y la diferenciación de subjetividades que impone la mercantilización de las relaciones sociales. Este discurso, acompañado de la pauperización de las condiciones materiales, se construyó eso que Gramsci denominó el sentido común con que se construye el propio terreno de disputa ${ }^{65}$. A través de esta interpelación directa al pueblo se apeló a representar la exclusión y la diferencia.

Por ejemplo, en palabras de Iglesias, Podemos es una opción real para que el poder político no sea un ejercicio privativo de una minoría privilegiada. Esta perspectiva se acerca a la gramática construida por el 15M, en tanto que no se trata de pedir a otros que tomen las decisiones, sino de tomarlas entre todos y todas. Para lograrlo, la estrategia política fue integral: "permear todos los espacios donde se construye y se crea comunidad; todos los espacios en los que es posible definir aspectos relevantes que afectan a la vida de las personas y la organización de nuestra vida común" ${ }^{66}$.

En función a la organización y al alcance del discurso, estos actores políticos lograron introducir algunos cambios disruptivos en su fase de consolidación, como un realineamiento del electorado y una recomposición general en la distribución del poder institucional. No obstante, la potente irrupción de Podemos en el panorama político español -elecciones europeas de 2014 y elecciones generales de 2015 y 2016-, su posición de poder todavía es asimétrica respecto a la estructura política tradicional. Los viejos partidos aún mantienen una mayoría parlamentaria y, por tanto, siguen determinando las principales decisiones ${ }^{67}$.

61 Inglehart, op. cit. 2001.

62 Oñate, op. cit. 2013.

63 Kitschelt. op.cit. 2006.

64 Las características básicas de los partidos cartel son: fines políticos auto-referenciales, una forma de entender la política como una profesión en sí misma, y dentro de una competencia política limitada. Vease Richard Katz y Peter Mair. "The cartel party thesis: A restatement". Perspectives on politics, 7:4, 2009, 753-766.

65 Gramsci, op. cit. 1975.

66 Iglesias, op. cit., 2015, 13.

67 Errejón, op.cit., 2015. 
En España, la irrupción de Podemos -y sus confluencias ${ }^{68}$ - condujo a un realineamiento de los votantes de izquierdas tras dos bloques con similar apoyo electoral, acabando de este modo con la dinámica bipartidista. En Chile, el Frente Amplio también avanzó en esta dirección, convirtiéndose en una fuerza política equiparable a la ex Nueva Mayoría en las elecciones presidencial y parlamentaria de 2017. Este resultado apalió la oligarquización del sistema político y la exclusión de los partidos políticos que no participaban de los grandes pactos ${ }^{69}$.

\subsection{Solidificación política de un quiebre generacional en la sociedad}

Podemos y el Frente Amplio son el resultado del cuestionamiento al consenso (neo)liberal en ambas sociedades. Esto confluyó con una mirada crítica del régimen político que emergió de las respectivas transiciones políticas. Este último aspecto no debe confundirse con una impugnación a la democracia, sino que al cierre excluyente del sistema político que impone la hegemonía ultra-liberal y limita los antagonismos. En esa contraposición, se presentaron como una alternativa al "consenso racional" del liberalismo económico y político ${ }^{70}$.

Ambas formaciones políticas diagnosticaron el agotamiento del marco político construido en las respectivas transiciones de dictadura a democracia. En tal sentido, Podemos cuestionó el Régimen del 78' o del turnismo, mientras que el Frente Amplio hizo lo mismo con el consenso transicional de los años $900^{\circ}$. Entienden aquella etapa como una auto-transformación del régimen autoritario y sus instituciones utiliza su propia legalidad para llevar adelante un cambio político, legitimado por el concurso de la oposición democrática de entonces y los posteriores gobiernos democráticos. Por ejemplo, para Íñigo Errejón, la transición española fue "una maniobra de restauración ampliada de la hegemonía, que oxigena el pluralismo e incorpora, en forma subordinada, a otros grupos sociales al bloque de poder [...] y fragua un relato y una intelectualidad que solidifican el nuevo orden" 71 . Desde esta premisa, Podemos planteó desde un comienzo una transformación del régimen político instaurado entre 1976 y 1982. Una mirada similar se planteó desde la dirigencia del Frente Amplio. En opinión de Gabriel Boric ${ }^{72}$, los pactos y alianzas políticas chilenas construidas por las élites de la transición, nunca se han organizado para favorecer a la población menos favorecida.

Esa mirada crítica de cada sociedad, que promovieron estos partidos-movimiento, es el resultado de un quiebre generacional. Esta es una afirmación que se sustenta en tres hechos que describen ambos procesos. En primer lugar, la juventud de sus principales dirigentes, que en su gran mayoría no superaban los 40 años de edad. Segundo, un discurso que se identificó desde un comienzo con los problemas que generaba la mercantilización de la vida en ambos países y que no habían sido atendidos por las generaciones políticas precedentes con intensidad ${ }^{73}$. Por último, el apoyo electoral que consiguieron provino, en gran medida, de los grupos etarios comprendido entre los 18 y los 45 años ${ }^{74}$.

68 En Comú Podem en Cataluña, Compromís en Comunidad Valenciana y en Marea en Galicia.

69 Eduardo Alvarado-Espina. "Exclusión, oligarquización y desafección política. Los efectos del sistema binominal en la democracia chilena", Enfoques, 13:53, 2015, 11-37.

70 Mouffe, op.cit., 2012.

71 Errejón y Mouffe, op. cit., 2015, 33

72 Boric, op. cit., 2017.

73 Carolina Álvarez Valdés y Antonia Garcés Sotomayor. La construcción de generación en los discursos juveniles del Chile actual, Revista Latinoamericana de Ciencias Sociales, Niñez y Juventud, 15:2, 2017, 991-1004.

74 Centro de Investigaciones Sociológicas (CIS). Barómetro Julio 2014. Estudio 3033. Madrid: CIS. 2014. Recuperado de: http://datos.cis.es/pdf/Es3033sd A.pdf; CADEM Plaza Pública. Track semanal de opinión pública del 1e de mayo de 2017. Estudio 174. Santiago de Chile. 2017. Recuperado de: https://plazapublica.cl/wp-content/uploads/archivos/Track-PP174Mayo-S2-VF.pdf. 
En síntesis, estas nuevas formaciones políticas tuvieron en la pauperización socioeconómica, que profundizó la desigualdad social, la fuerza de empuje necesaria para articular un nuevo discurso de hegemonía. Ello abrió un espacio a las opciones políticas de ruptura de carácter contra-hegemónicas. Entre estas opciones, cabe destacar las de cambiar las reglas del juego que definen la relación entre política y sociedad desde una observación jerárquica, y enfrentar el discurso neoliberal relativo al modelo socioeconómico. A simple vista, puede parecer paradójico que dos países de renta media-alta y con escasa cultura cívica puedan dar cabida a organizaciones políticas radicales que cuestionan el funcionamiento del régimen político y el sistema social. No obstante, las tensiones entre la política y lo político, y la dificultad para expresar la diferencia y la exclusión, propia de la configuración política tradicional, crearon un contexto adecuado para la emergencia de partidos movimiento que impulsaran la subversión política. En la acera contraria, la política tradicional se mostró impotente para introducir los cambios necesarios para superar las condiciones de desigualdad y exclusión de ambos países.

Tabla 2. Principales convergencias y divergencias entre Podemos (P) y el Frente Amplio (FA)

\begin{tabular}{|c|c|c|c|}
\hline Dimensión & $\begin{array}{l}\text { Aspectos que marcan la emergencia y el carácter disruptivo } \\
\text { de los nuevos actores políticos de izquierdas }\end{array}$ & $\mathbf{P}$ & FA \\
\hline \multirow[t]{2}{*}{ Socioeconómica } & $\begin{array}{l}\text { Alta desigualdad de ingresos o notable aumento de ésta en el } \\
\text { respectivo país }\end{array}$ & $\mathrm{Si}$ & $\mathrm{Si}$ \\
\hline & Bajo gasto social o disminución del mismo en el respectivo país & $\mathrm{Si}$ & $\mathrm{Si}$ \\
\hline \multirow{3}{*}{ Político-cultural } & $\begin{array}{l}\text { Movimiento social que rompe el consentimiento político previo } \\
\text { otorgado al sistema político y sus élites }\end{array}$ & $\mathrm{Si}$ & $\mathrm{Si}$ \\
\hline & $\begin{array}{l}\text { Percepción de que la política está controlada por poderosos } \\
\text { agentes económicos }\end{array}$ & $\mathrm{Si}$ & $\mathrm{Si}$ \\
\hline & $\begin{array}{l}\text { Aumento de la politización de la sociedad (mayor participación } \\
\text { electoral) }\end{array}$ & $\mathrm{Si}$ & No \\
\hline \multirow{5}{*}{ Génesis } & Liderazgos reconocibles para la población & $\mathrm{Si}$ & $\mathrm{Si}$ \\
\hline & $\begin{array}{l}\text { Se presentan como una alternativa al "duopolio" político que ha } \\
\text { gobernado en el país desde la transición }\end{array}$ & $\mathrm{Si}$ & $\mathrm{Si}$ \\
\hline & $\begin{array}{l}\text { Marcado discurso contra-hegemónico basado en el clivaje } \\
\text { élites/pueblo }\end{array}$ & $\mathrm{Si}$ & $\mathrm{Si}$ \\
\hline & Amplia organización desde abajo & $\mathrm{Si}$ & No \\
\hline & $\begin{array}{l}\text { Consolidación como fuerza política alternativa al bipartidismo } \\
\text { antes de unas elecciones generales }\end{array}$ & $\mathrm{Si}$ & No \\
\hline \multirow{5}{*}{$\begin{array}{l}\text { Estrategia, } \\
\text { proyecto y } \\
\text { consolidación }\end{array}$} & Identificación con los valores postmateriales & $\mathrm{Si}$ & $\mathrm{Si}$ \\
\hline & Rechazo al encasillamiento en el eje izquierda/derecha & $\mathrm{Si}$ & $\mathrm{Si}$ \\
\hline & Propuestas para un nuevo proceso constituyente & $\mathrm{Si}$ & $\mathrm{Si}$ \\
\hline & Realineamiento de los votantes de izquierdas & $\mathrm{Si}$ & $\mathrm{Si}$ \\
\hline & Desafío al bipartidismo/bipartismo & $\mathrm{Si}$ & Parcial \\
\hline
\end{tabular}

Fuente: elaboración propia.

\section{Conclusiones}

En este artículo se han analizado las condiciones sociales y políticas que condujeron, tanto en España como en Chile, a la emergencia de nuevos partidos políticos. El conjunto de antecedentes teóricos y de fuentes de información secundaria relevante que se han analizado, nos ha permitido explicar, a modo comparativo, la relación de antagonismos y aspiraciones hegemónicas que dieron sustento a una nueva construcción política desde los movimientos sociales del año 2011 en ambos países. Para tal efecto, se 
fijaron cuatro dimensiones centrales de análisis, de las cuales podemos concluir lo siguiente.

Primero, y considerando que las trayectorias socioeconómicas de Chile y España en los últimos cuarenta años han sido diferentes, en ambos casos los movimientos sociales plantearon un desafío al régimen político, empujado por una realidad socioeconómica que difiere de las expectativas de bienestar, justicia y seguridad que tiene la ciudadanía. En la práctica, se constata un quiebre en el contrato social tácito defendido en las transiciones post-autoritarias.

Segundo, a partir de este primer problema, se resquebraja el consentimiento que existía hacia el discurso político hegemónico, profundizándose la desconfianza hacia las élites políticas gobernantes. Esto abre un espacio para la emergencia de un relato contra-hegemónico o -parafraseando a Gramsciun nuevo sentido común que representa a los excluidos del sistema. Esto produce un debilitamiento en el imperio del consenso racional original con el que se construyó el relato de las élites políticas en ambas democracias. Un efecto constatable de este proceso es su correlación con una mayor movilización y realineamiento electoral y la instalación de nuevos actores políticos como una tercera fuerza política a nivel nacional o estatal.

Una tercera conclusión hace referencia al modo en que se posicionan y asientan los proyectos políticos de Podemos y el Frente Amplio. Dentro de las similitudes se observa un discurso claramente asociado a un relato contra-hegemónico basado en la dicotomía pueblo versus élite, donde ambos proyectos políticos apelaron a la construcción de un nuevo sentido común -los de arriba frente a los de abajo; y, por otra parte, una identificación con los valores postmateriales -igualdad de género, derechos de las minorías y excluidos, protección del medio ambiente, tolerancia y no discriminación. Entre las diferencias, se ha identificado que la organización de cada partido-movimiento, aunque se sostiene en la convergencia de diversos partidos minoritarios de izquierdas en ambos países, su consolidación e impacto no es el mismo. Podemos está presente en casi todo el territorio español, gracias a un proceso autoconvocado de ciudadanos y organizaciones sociales. Esto le ha permitido desafiar seriamente, en las elecciones municipales, autonómicas y generales, al bipartidismo imperfecto que ha gobernado en España desde la transición. En cambio, el Frente Amplio aún no representa una alternativa empírica al duopolio político chileno, aunque si tiene un apoyo electoral relativamente cautivo.

Una cuarta conclusión apunta a que las estrategias y el proyecto político de ambos partidosmovimiento han conseguido presentarse, en un primer momento, como una alternativa disruptiva $-\mathrm{y}$ con un toque subversivo- al statu quo que ofrecía el juego político asentado en las transiciones a la democracia, desde el rechazo al eje clásico de diferenciación ideológica izquierda/derecha hasta la solidificación de un espacio político enfrentado al bipartidismo. Además, que sean los jóvenes quienes otorgan más soporte electoral a estos conglomerados viene a confirmar ese cambio en las preferencias políticas.

De acuerdo a estas conclusiones, se puede inferir que una respuesta al interrogante desde el cual se plantea este análisis es que en ambos países se dieron condiciones, tanto estructurales como culturales, que facilitaron la cristalización de las propuestas de los movimientos sociales en partidos-movimientos. Por lo tanto, la consolidación política e institucional de estos partidos no se debió a un mero clima de malestar o dentro de una determinada coyuntura. Aunque el ensayo constante de estas transformaciones es uno de los ejercicios sustantivos del quehacer político, no se pueden dejar de considerar las altas probabilidades de consolidación que brinda el contexto socioeconómico y cultural global.

Ahora bien, a partir del análisis también emerge la pregunta respecto a la real consolidación que estos partidos pueden tener en el tiempo -sobre todo el Frente Amplio- como fuerzas que puedan ofrecer una alternativa al bipartidismo. Si acaso se lograran plasmar en la praxis, los discursos, las declaraciones de principios y su programa, lo que sin duda planteará desafíos contingentes en la disputa del poder y el diseño institucional.

Incluso, en un contexto más amplio, podría plantearse otro par de interrogantes respecto a la consolidación de este tipo de partidos, los cuales claramente exceden los límites y pretensiones de este trabajo: ¿no será acaso que estos movimientos políticos son uno de los síntomas de -y una respuesta a- 
una tensión que sitúa a los regímenes políticos de la modernidad en un proceso de transformación de más largo aliento? y jla construcción de la nueva hegemonía podría generar un espacio de resistencia a la praxis antidemocrática neoliberal?

\section{Referencias Bibliográficas}

\section{Fuentes impresas}

Eduardo Alvarado Espina. "Una aproximación crítico-contextual al declive de la democracia en la era neoliberal", Revista Española de Ciencia Política, 47, 2018, 69-91.

Eduardo Alvarado Espina. "Exclusión, oligarquización y desafección política. Los efectos del sistema binominal en la democracia chilena”, Enfoques, 13:22, 2015, 11-37.

Carolina Álvarez Valdés y Antonia Garcés Sotomayor. La construcción de generación en los discursos juveniles del Chile actual, Revista Latinoamericana de Ciencias Sociales, Niñez y Juventud, 15:2, 2017, 991-1004.

Gabriel Boric. "Los movimientos sociales y la política de hoy: una mirada con perspectiva histórica", Pablo Rivera-Vargas, Elena Sánchez, Rommy Morales-Olivares, (coords). Conocimiento para la equidad social: pensando Chile globalmente. Santiago de Chile, Colección Políticas Públicas USACH, 2016, 250-256.

Manuel Castells. Redes de indignación y esperanza, Madrid, Alianza, 2012.

Jacques Derrida. El otro cabo. La democracia, para otro día. Colección Delos. Ediciones del Serval. Barcelona.

Carlos Durán, "Campo político-institucional y procesamiento del malestar social en Chile, 1999-2009", Izquierdas, 40, 2018, 1-32.

Íñigo Errejón, "PODEMOS como práctica cultural emergente frente al imaginario neoliberal: hegemonía y disidencia. Conversación con Íñigo Errejón”, IC Revista Científica de Información y Comunicación, 11, 2015, 17-46.

Ínigo Errejón y Chantal Mouffe. Construirpueblo. Hegemonía y radicalización de la democracia, Barcelona, Icaria, 2015.

Gøsta Esping-Andersen. Politics against markets: The social democratic road to power, Princeton, NJ, Princeton University Press, 1985.

Ricardo Ffrench-Davis. Entre el Neoliberalismo y el Crecimiento con Equidad: Tres De $\square$ cadas de Pol $\square$ tica Econo $\square$ mica en Chile, Santiago de Chile, LOM Ediciones, 2003.

Cristina Flesher y Laurence Cox, Understanding European movements: New social movements, global justice struggles. London, UK, Routledge, 2013.

Arturo Fontaine. "El miedo y otros escritos: el pensamiento de Jaime Guzmán”, en Estudios Públicos, 42, 1991.

Manuel Antonio Garreto $\square$ n. La gran ruptura: Institucionalidad politica y actores sociales en el Chile del siglo XXI. Santiago, LOM ediciones, 2016.

Juan Jesús González. "Crisis de la democracia de partidos y segunda transición", Revista de Derecho Politico, 100, 2017, 615-638.

Antonio Gramsci. Cartas desde la cárcel, Madrid, Cuadernos para el diálogo, 1975.

Isabel Hinojosa. "La ideología franquista en la legitimación de la dictadura militar chilena", Revista Complutense de Historia de América, 34, 2008, 233-250.

Patricia Hipsher. "Democratization and the decline of urban social movements in Chile and Spain", en Comparative Politics, 28:3, 1996, 273-297.

Eric Hobsbawm. Guerra y paz en el siglo XXI, Barcelona, Crítica, 2007.

Pablo Iglesias, "Understanding Podemos", in New Left Review, 93, 2015, 7-22. 
Pablo Iglesias, Íñigo Errejón, Juan Carlos Monedero, Carolina Bescansa y Luis Alegre. Borrador de Principios Políticos, Madrid, Podemos. 2014. Recuperado de: goo.gl/C0p52D

Ronald Inglehart. Modernización y posmodernización: el cambio cultural, político y económico en 43 sociedades, Madrid, CIS. 2001.

Ronald Inglehart. Silent Revolution: Changing Values and Political Styles among Westerns Publics. New Jersey, Princeton University Press, 1977.

Bob Jessop. Nicos Poulantzas: Marxist Theory and Political Strategy. Londres, McMillan, 1985.

Richard Katz y Peter Mair. “The cartel party thesis: A restatement”. Perspectives on politics, 7:4, 2009, 753766.

Herbert Kitschelt. "Movement parties". Handbook of party politics, 1, 2006, 278-290.

Ernesto Laclau, La Razón populista, Buenos Aires, FCE, 2005.

Ernesto Laclau, Deconstrucción, pragmatismo y hegemonía. En Chantal Mouffe. Comp. Deconstrucción y Pragmatismo. Buenos Aires. Paidós. 1998.

Bernard Manin. Los principios del Gobierno Representativo. Madrid, Alianza, 2010.

Juan Carlos Monedero. "¿Posdemocracia? Frente al pesimismo de la nostalgia, el optimismo de la desobediencia”, Nueva Sociedad, 240, julio-agosto, 2012.

Wolfgang Merkel. "Is capitalism compatible with democracy?", in Zeitschrift für Vergleichende Politikwissenschaft, 8:2, 2014, 109-128.

Rommy Morales-Olivares. "Inconsistencies between social-democratic discourses and neo-liberal institutional practices in Chile and South Africa: a comparative analysis of the post-authoritarian periods". Peter Wagner, P. (Ed). African, American and European trajectories of modernity, in Edinburgh, Edinburgh University Press, 2015, 125-148

Chantal Mouffe. Desconstrucción y pragmatismo. Buenos Aires, Paidón, 1998.

Chantal Mouffe. La Paradoja Democrática: El Peligro del Consenso en la Política Contemporánea, Barcelona, Gedisa, 2012.

Vicenç Navarro. "El error de las políticas de austeridad, en la sanidad pública", Gaceta Sanitaria, 26: 2, 2012, 174-175.

Pablo Oñate, "La movilización ciudadana en España en los albores del siglo XXI: una contextualización para el debate", Revista Española de Ciencia Política, 33, 2013, 31-55.

Thomas Piketty, El capital en el siglo XXI. Barcelona, RBA, 2015.

Patricio Rozas y Gustavo Marín, “1988: ‘el mapa de la extrema riqueza' 10 años después”, Santiago de Chile, CESOC, 1988.

Pablo Rivera-Vargas, "Modernización Reflexiva en Chile: Participación Electoral y Confianza de la Ciudadanía en las Instituciones durante la Post Dictadura: Análisis entre los Años 1999-2010”, Politicas Públicas, 9:2, Santiago, Universidad de Santiago, 2016, 90-108.

Sidney Tarrow. El poder en movimiento. Los movimientos sociales, la acción colectiva y la política. Madrid, Alianza, 1997.

Luis Thielemann. Para una periodificación del Movimiento Estudiantil de la transición (1987-2011). Revista Pretérito Imperfecto, 2011.

Alain Touraine y Pablo Rivera-Vargas. "Actores, conflictos y reformas en sociedades de comunicación global”. P. Rivera-Vargas, E. Sánchez, R. Morales-Olivares. (coords). Conocimiento para la equidad social: pensando Chile globalmente. Santiago de Chile, Colección Políticas Públicas - USACH. 2017, $33-40$

Manuel Villoria y Fernando Jiménez. "La corrupción en España (2004-2010): datos, percepción y efectos”, Revista Española de Investigaciones Sociológicas (REIS), 138:1, 2012, 109-134.

Sheldon S. Wolin. Democracia S. A. La democracia dirigida y el fantasma del totalitarismo invertido. Madrid, Katz editores, 2008. 


\section{Otras fuentes}

World Values Survey. Wave 6, 2010-2014. Viena, Institute for Comparative Survey Research, 2014. Recuperado de: https://goo.gl/Cz74Bd

Latinobarómetro. Informe 2013, Santiago de Chile, Corporación Latinobarómetro, 2013. Recuperado de http://www.latinobarometro.org/latOnline.jsp.

OCDE, ¿Cómo va la vida en España? Better Life Iniciative. Paris: OCDE, 2016, Recuperado de: goo.gl/FXHbWJ

CADEM Plaza Pública. Track semanal de opinión pública del 1e de mayo de 2017. Estudio 174.

Santiago de Chile. 2017. Recuperado de: https://plazapublica.cl/wpcontent/uploads/archivos/Track-PP174-Mayo-S2-VF.pdf.

Centro de Investigaciones Sociológicas (CIS). Barómetro Mayo 2014. Estudio 3024. Madrid: CIS. 2014. Recuperado de: http://datos.cis.es/pdf/Es3024mar A.pdf

Centro de Investigaciones Sociológicas (CIS). Barómetro Julio 2014. Estudio 3033. Madrid: CIS. 2014. Recuperado de: http://datos.cis.es/pdf/Es3033sd A.pdf.

Parlamento Europeo. Resultado elecciones europeas de 2014, 2014. Recuperado de: goo.gl/hjhXi3 